\title{
WEAK TANGENT CONES AND OPTIMIZATION IN A BANACH SPACE*
}

\section{J. BORWEIN $\dagger$}

\begin{abstract}
A general notion of a $\tau$-tangent cone is introduced and developed for optimization purposes. This includes as special cases both the weak and strong tangent cones that appear in the literature.

First order conditions with and without constraint qualification are examined and particular examples are provided to demonstrate that these conditions properly subsume those previously in the literature. Emphasis is placed on weak Kuhn-Tucker sufficiency conditions.
\end{abstract}

1. Introduction. Suppose that $X$ and $Y$ are real Banach spaces and that $f: X \rightarrow R$, $g: X \rightarrow Y$ are Fréchet differentiable at $\bar{x}$. Let $(\mathrm{P})$ denote the general program

$$
\text { minimize } f(x) \text { subject to } g(x) \in B, \quad x \in C,
$$

where $B \subset X, C \subset Y$ are arbitrary sets. $(\mathrm{P})$ is presumed when necessary conditions are discussed for a program to assume a minimum at $\bar{x}$.

This general programming problem, which includes the standard Kuhn-Tucker program [17], has been studied by Varaiya [25], Guignard [12], Zlobec and Massam [28] and others. These authors use the notion of a tangent cone, introduced by Abadie [1], which extends the Kuhn-Tucker notion of a feasible direction.

In this paper the concept of a $\tau$-tangent cone is applied to produce first order necessary and sufficient optimality conditions for $(\mathrm{P})$ analogous to those in [4], [12], [27]. Examples are given to show that in both directions these are stronger than previous results. Fritz John conditions are also considered.

2. Preliminaries. For any two topological spaces $X$ and $Y, L[X, Y]$ will denote the continuous, linear mappings between $X$ and $Y$. For any locally convex real topological vector space $X, X^{\prime}$ denotes $L[X, R]$ which will be given the weak* topology $\sigma\left(X^{\prime}, X\right)$. When $M$ is a subset of $X$ or $X^{\prime}, M^{0}, \bar{M}$ and [M] denote respectively the interior, closure and closed convex hull of $M$ (in the appropriate topology). Convergence in norm is denoted $\rightarrow$ while convergence in another linear topology $\tau$ is denoted $\rightarrow$ or $\rightarrow(\tau) . N(T)$ and $R(T)$ denote the null space and range of a linear operator $T . T^{*}$ denotes the adjoint of $T$.

Definition 1. $C \subset X\left(C^{\prime} \subset X^{\prime}\right)$ is a cone if for $x \in C\left(x^{\prime} \in C^{\prime}\right), \alpha x \in C\left(\alpha x^{\prime} \in C^{\prime}\right)$ for all $\alpha \geqq 0$.

Definition 2. (i) For a cone $C \subset X$, one defines

$$
C^{+}=\left\{x^{\prime} \in X^{\prime}: x^{\prime}(x) \geqq 0 \text { for all } x \in C\right\} .
$$

( $C^{+}$is called the dual cone of $C$ and is always closed and convex.)

(ii) For a cone $C^{\prime} \in X^{\prime}$, one defines

$$
C^{++}=\left\{x \in X: x^{\prime}(x) \geqq 0 \text { for all } x^{\prime} \in C^{\prime}\right\} .
$$

$C^{-}=-\left(C^{+}\right)=(-C)^{+}$. Similarly $\left(C^{\prime}\right)^{-}=\left(-C^{\prime}\right)^{+}$.

Definition 3 [15]. Let $f: X \rightarrow R \cup\{\infty\}$ be a convex function. The subgradient set of $f$ at $x$, denoted $\partial f(x)$, is the set of vectors in $X^{\prime}$ satisfying

$$
x^{*}(y-x) \leqq f(y)-f(x) \quad \forall y \in X .
$$

\footnotetext{
* Received by the editors December 11, 1975, and in revised form June 30, 1977.

$\dagger$ Department of Mathematics, Dalhousie University, Halifax, Nova Scotia, Canada B3H 3T5. This work was supported in part by the National Research Council of Canada under Account A4493.
} 
If we let $i(x \mid A)$ denote the indicator function of a set $A(i(x \mid A)=0$ if $x \in A$, $i(x \mid A)=\infty$ if $x \notin A)$ we then have the relationship

$$
\partial i(0 \mid C)=C^{-} \text {. }
$$

Proposition 1. Suppose $C, D$, are convex cones in a locally convex space $X$ and that $C^{\prime}$ is a convex cone in $X^{\prime}$.

(i) $\left(C^{+}\right)^{+}=\bar{C} ;\left(G^{\prime}\right)^{++}=\overline{C^{\prime}}$.

(ii) $(\bar{C} \cap \bar{D})^{+}=\bar{C}^{+}+D^{+}$.

Proof. These are proved in normal spaces in [21]. The extensions offer no problems.

Remark. Guignard [12] claims (i) and (ii) with the norm topology on $X^{\prime}$. This is only true when $X$ is reflexive. As an example consider $X=l_{1}$ and the sequence space $c_{0}$ as a (closed) cone in $l^{\infty}$. Then $c_{0}$ is norm closed in $l^{\infty}$ but $c_{0}$ is $\sigma\left(l^{\infty}, l_{1}\right)$ dense in $l^{\infty}$. Thus $\left(c_{0}\right)^{++}=l^{\infty}$.

Proposition 2. Suppose that $C$ and $D$ are closed cones is a locally convex space $X$ and that $C^{0} \cap D \neq \varnothing$. Then

$$
(C \cap D)^{+}=C^{+}+D^{+}
$$

Proof. This is a consequence of the subgradient relationship $\partial f(x)+\partial g(x)=$ $\partial(f+g)(x)$ applied to $f(x)=i(X \mid C)$ and $g(x)=i(X \mid D)$ which holds with the hypothesis $C^{0} \cap D \neq \varnothing$. The general relationship is proved by Rockafellar in [24].

Ritter has proved a similar result in [21] for normed spaces.

Definition 4 [25]. $h$ is tangent to $A$ at $\bar{x}$ if there is a sequence $\left\{x_{n}\right\}$ in $A$ with $x_{n} \rightarrow \bar{x}$ and a sequence $\left\{\lambda_{n}\right\}$ of nonnegative real numbers with $\lambda_{n}\left(x_{n}-\bar{x}\right) \rightarrow h$. The set $T(A, \bar{x})$ of all tangents to $A$ at $\bar{x}$ is called the tangent cone to $A$ at $\bar{x}$.

Definition 5 [12]. $P(A, \bar{x})$, the closed convex hull of $T(A, \bar{x})$, is called the pseudotangent cone to $A$ at $\bar{x}$.

Let $\tau$ denote another locally convex (Hausdorff) topology on $X$ which is (1) coarser than the norm topology $s$ and satisfies the property (2) that $\tau$-convergent sequences are $s$-bounded. Property (2) is always satisfied by any topology of the dual pair $\left(X, X^{\prime}\right)[22$, Thm 1, p. 67] and so in particular by the weak topology $\sigma$. If $X$ is the dual of a Banach space it is also true of the weak* topology $\sigma^{*}[22$, Cor. 1, p. 66]. We can now introduce the following notions.

Definition 6 [4]. We say the vector $h$ is a $\tau$-tangent to $A$ at $\bar{x}$ if there is a sequence $\left\{x_{n}\right\}$ in $A$ with $x_{n} \rightarrow \bar{x}$ and a sequence $\left\{\lambda_{n}\right\}$ of nonnegative real numbers such that $\lambda_{n}\left(x_{n}-\bar{x}\right) \rightarrow(\tau) h . T_{\tau}(A, \bar{x})\left(\subset T_{\tau}([A], \bar{x})\right)$ which will be called the $\tau$-tangent cone to $A$ at $\bar{x}$, consists of all such vectors $h$.

Definition 7. $P_{\tau}(A, \bar{x})$ will denote the closed convex hull of $T_{\tau}(A, \bar{x})$ and will be called the $\tau$-pseudotangent cone to $A$ at $\bar{x}$.

Remarks. (i) Thus $T(A, x)=T_{s}(A, x)$ and $P(A, x)=P_{s}(A, x)$. In this case we will generally drop the subscript.

(ii) It is clear that if $\bar{x} \in \bar{A}$ then $0 \in T(A, \bar{x})$, and that if $\bar{x} \in A^{0}$ then $T(A, \bar{x})=X$.

(iii) Since $P_{\tau}(A, \bar{x})$ is norm closed and convex it is also weakly closed.

(iv) The tangent cone is always a closed cone, but need not be convex. $T_{\tau}(A, \bar{x})$ need not, in general, be closed.

(v) It is clear that as $\tau$ gets weaker the corresponding cones increase.

Definition 8. $A_{1}$ is said to be $\tau$-pseudoconvex with respect to $A_{2}$ at $\bar{x}$ when $A_{1}-\bar{x} \subset P_{\tau}\left(A_{2}, \bar{x}\right)$. When $A_{1}=A_{2}, A_{1}$ is simply said to be $\tau$-pseudoconvex at $\bar{x}$. 
This final case with $\tau=s$ corresponds to Guignard's definition of pseudoconvexity. Again we will often drop the $s$. It is easy to verify that if there is some set $S$, with $A_{1} \subset S \subset A_{2}$, such that $S$ is starshaped at $\bar{x}$ then $A_{1}$ is pseudoconvex with respect to $A_{2}$ at $\bar{x}$. In particular, any closed convex set is pseudoconvex at all its members. The set $A=\{1 / n\}_{n=1} \bigcup\{0\}$ is a simple example of a disconnected set which is pseudoconvex at 0 . From now on it is supposed that $\bar{x} \in \bar{A}$.

Proposition 3. Suppose $\tau$ is a topology of the dual pair. Then

(i) $T(A, \bar{x}) \subset T_{\tau}(A, \bar{x}) \subset T_{\tau}([A], \bar{x})=T([A], \bar{x})=P([A], \bar{x})$.

(ii) If $A$ is $\tau$-pseudoconvex at $\bar{x}$ then $P_{\tau}(A, \bar{x})=P([A], \bar{x})$.

Proof. (i) The first two containments are immediate. The final equality follows from $T([A], \bar{x})=\bar{U}_{t \geqq 0} t([A]-\bar{x})$ (see [25]). Moreover, since $\bigcup_{t \geqq 0} t([A]-\bar{x})$ is convex, it has the same weak and norm closures, and thus $T_{\tau}([A], \bar{x})=T([A], \bar{x})$. (ii) When $A$ is $\tau$-pseudoconvex at $\bar{x}$ one has $A-\bar{x} \subset P_{\tau}(A, \bar{x})$. Since this last set is convex and closed $\bigcup_{t \geqq 0} t([A]-\bar{x}) \subset P_{\tau}(A, \bar{x})$. In conjunction with $P_{\tau}(A, \bar{x}) \subset P([A], \bar{x})$, this proves (ii).

The next proposition generalizes a result in Rockafellar [24] from convex to $\tau$-pseudoconvex sets.

Proposition 4. Suppose that $\tau$ is a topology of the dual pair and that the following conditions hold:

(i) $[A] \cap[B]=[A \cap B]$,

(ii) $[A]^{0} \cap[B]^{0} \neq \varnothing$,

(iii) $A \cap B$ is $\tau$-pseudoconvex at $\bar{x}$.

Then $P_{\tau}(A, \bar{x}) \cap P_{\tau}(B, \bar{x})=P_{\tau}(A \cap B, \bar{x})$.

Proof. It clearly suffices to show that $P_{\tau}(A ; \bar{x}) \cap P_{\tau}(B, \bar{x}) \subset P_{\tau}(A \cap B, \bar{x})$. By (i) and Proposition 3 (ii) we have

$$
P_{\tau}(A \cap B, \bar{x})=P_{\tau}([A \cap B], \bar{x})=P([A] \cap[B], \bar{x}) .
$$

However, $P([A] \cap[B], \bar{x})=P([A], \bar{x}) \cap P([B], \bar{x})$ since (ii) holds. This is proved in $R^{n}$ in [24] and the proof is unchanged. Since $P([A], \bar{x})$ contains $P_{\tau}(A, \bar{x})$ and $P([B], \bar{x})$ contains $P_{\tau}(B, \bar{x})$ we have the required containment.

All the conditions are necessary, in that easy examples exist in $R^{2}$ which satisfy any two of the hypotheses and not the conclusion.

Borwein and O'Brien [6] have examined a variety of tangent cone properties. In particular, they have shown that a weakly compact set in a normed space which is pseudoconvex at all its points is convex. This can be extended using a recent result of Lau's [18] to show that whenever $A$ is a closed set in a reflexive space

$$
\bigcap\{P(A, a)+a: a \in A\}=\operatorname{star} A
$$

where star $A=\{\bar{a} \in A: t \bar{a}+(1-t) a \in A$ for $0 \leqq t \leqq 1, a \in A\}$. This, in conjunction with requiring that $A$ is pseudoconvex at all its members, now implies that $A$ is convex.

In general the notion of $\tau$-pseudoconvexity is strictly weaker than that of pseudoconvexity as the next example shows.

Example 1. Let $X=C[0,1]$, the continuous functions with sup norm. Then $A=\{f: f \in C[0,1],\|f\|=1, f(x)=1$ for some $x \in[0,1]\}$ is weakly pseudoconvex at all $a \in \bar{A}$ and is not pseudoconvex at some $a \in \bar{A}$.

Proof. Obviously $A$ is norm closed. To show that it is weakly pseudoconvex, we fix $f$ in $A$ and show that for any other $g$ in $A, g-f$ is in $T_{\sigma}(A, f)$.

Since for some $x_{0}$ in $[0,1] f\left(x_{0}\right)=1$, there exist points $x_{m}^{1}, x_{m}^{2}$ in $[0,1]$ with $x_{m}^{1}<x_{m}^{2}, x_{0} \notin\left[x_{m}^{1}, x_{m}^{2}\right], x_{m}^{1} \rightarrow x_{0}$ and $x_{m}^{2} \rightarrow x_{0}$ and such that $f(y)>1-(1 / m)$ for all 
$y \in\left[x_{m}^{1}, x_{m}^{2}\right]$. Set $k_{m}(x)=((m-1) / m) f(x)+(1 / m) g(x)$ and

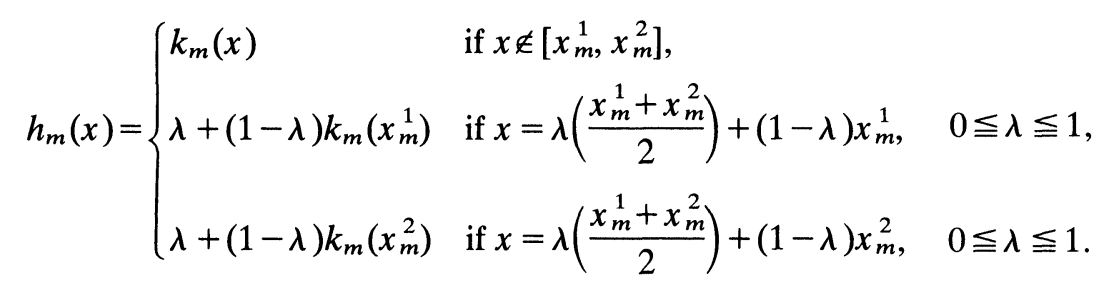

By construction $h_{m} \in A$ and

$$
\left\|f-h_{m}\right\|<\frac{2}{m}, \quad \text { so } \quad h_{m} \rightarrow f \quad \text { in norm. }
$$

Also for any $x \notin\left[x_{m}^{1}, x_{m}^{2}\right], m\left(k_{m}(x)-f(x)\right)=g(x)-f(x)$ so $m\left(h_{m}-f\right) \rightarrow g-f$ pointwise. Since $\left\|m\left(h_{m}-f\right)\right\|<2$ for all $m$, the Lebesgue bounded convergence theorem implies $\mu\left(m\left(h_{m}-f\right)\right) \rightarrow \mu(g-f)$ for any continuous linear functional $\mu$ on $C[0,1]$. Thus $m\left(h_{m}-f\right)$ converges weakly to $g-f$ and $g-f \in T_{\sigma}(A, f)$.

It remains to show that $A$ is not pseudoconvex. It is easy to do this by showing that if $i(x)=x$ is the identity function on $[0,1]$, then $P(A, i) \subset$ $\{\lambda(g-i): g \in A, g(1)=1\}$. Thus $A$ is not pseudoconvex at $i$.

So at least in a nonreflexive Banach space, a bounded weakly pseudoconvex set need not be convex.

Definition 9. $f: X \rightarrow R$ is quasiconvex on a convex set $C$ if

$$
\{x \in X: x \in C, f(x) \leqq r\}
$$

is convex for all $r \in R$.

Definition 10. $f: X \rightarrow R$ is pseudoconvex on a set $A$ at $\bar{x}$ if $f$ is Fréchet differentiable and if $x \in A, f^{\prime}(\bar{x})(x-\bar{x}) \geqq 0$ implies $f(x) \geqq f(\bar{x})$.

The next proposition gives a simple relation between these two concepts. It generalizes and observation of Guignard [12].

Proposition 5. Suppose $f: X \rightarrow R$ is quasiconvex on a convex set $C$ and differentiable. Suppose that, for some $y \in C, f^{\prime}(\bar{x})(y-\bar{x})>0$. Then $f$ is pseudoconvex at $\bar{x}$ on $C$.

Proof. Suppose $f^{\prime}(\bar{x})(x-\bar{x}) \geqq 0 \forall x \in C$. Then, for $0<\alpha \leqq 1,(1-\alpha) f^{\prime}(\bar{x})(x-\bar{x})+$ $\alpha f^{\prime}(\bar{x})(y-\bar{x})>0 \forall x \in C$. Since $x, y \in C$ and $C$ is convex $x_{\alpha}=(1-\alpha) x+\alpha y \in C$ and $f^{\prime}(\bar{x})\left(x_{\alpha}-\bar{x}\right)>0$. Since $f$ is supposed quasiconvex on $C$ it follows easily that $f\left(x_{\alpha}\right)>$ $f(\bar{x})$ for $0<\alpha \leqq 1$. Since $f$ is continuous, $f(x) \geqq f(\bar{x})$.

Guinard uses this lemma in the case that $C=X$ and $f^{\prime}(\bar{x}) \neq 0$.

Definition 11. $g: X \rightarrow Y$ is $\tau_{1}-\tau_{2}$ continuous if it is continuous from the $\tau_{1}$ topology on the $X$ to the $\tau_{2}$ topology on $Y$. If $\tau_{1}=\sigma$ and $\tau_{2}=s$ we say $g$ is completely continuous.

The complete continuity of $g$ implies that of $g^{\prime}(\bar{x})$ (on bounded sets) [26]. Since $g^{\prime}(\bar{x})$ is linear and continuous it is completely continuous when it is compact (maps bounded sets into compact sets). For simplicity in many of the following results one may assume compactness of $g^{\prime}(\bar{x})$ in cases in which complete continuity suffices. This in particular insures that $g^{\prime}(\bar{x})$ is $\tau-s$ continuous whenever $\tau$ is a dual topology.

3. First order optimality conditions. Consider (P). Following Guignard [12], let $\Delta$ denote $\{x: g(x) \in B\}$, and let $A$ denote the feasible set $\Delta \cap C$. The first proposition of this section gives a $\tau$-tangent cone containment analogous to the one proven in [12].

Proposition 6. Suppose that $g^{\prime}(\bar{x})$ is $\tau_{1}-\tau_{2}$ continuous. Then

$$
g^{\prime}(\bar{x})\left(P_{\tau_{1}}(\Delta, \bar{x})\right) \subset P_{\tau_{2}}(B, g(\bar{x})) \text {. }
$$


Proof. Suppose $h$ is nonzero and belongs to $T_{\tau_{1}}(\Delta, \bar{x})$. Then there is a sequence $\left\{h_{n}\right\}, h_{n}=\lambda_{n}\left(x_{n}-\bar{x}\right) \rightarrow\left(\tau_{1}\right) h$, with $\left\{x_{n}\right\} \subset A, x_{n} \rightarrow \bar{x}$ and $\lambda_{n} \geqq 0$ for all $n \in N$. Since $h \neq 0$, one may assume that $\lambda_{n} \rightarrow \infty$ and $\lambda_{n} \geqq 1$. $g$ is supposed Fréchet differentiable at $\bar{x}$. It follows that

$$
\frac{g(\bar{x}+t k)-g(\bar{x})}{t}-g^{\prime}(\bar{x})(x) \rightarrow 0,
$$

uniformly on bounded sets in $X$, as $t \rightarrow 0$. In particular, $\left\{h_{n}\right\}$, being a $\tau_{1}$-convergent sequence, is bounded by assumption (1) on $\tau_{1}$. Thus

$$
\frac{g\left(\bar{x}+\lambda_{n}^{-1} h_{n}\right)-g(\bar{x})}{\lambda_{n}^{-1}}-g^{\prime}(\bar{x})\left(h_{n}\right) \rightarrow 0
$$

as $n \rightarrow \infty$. Moreover, $g^{\prime}(\bar{x})$ is, by hypothesis, $\tau_{1}-\tau_{2}$ continuous. It follows that $\lambda_{n}(g(\bar{x}+$ $\left.\lambda_{n}^{-1} h_{n}\right)-g(\bar{x})$ ) converges $\tau_{2}$ to $g^{\prime}(\bar{x})(h)$. (This uses assumption (2).) Since $\bar{x}+\lambda_{n}^{-1} h_{n}=$ $x_{n} \in \Delta$, and $x_{n} \rightarrow \bar{x}$ one has $g\left(x_{n}\right) \in B$ and $g\left(x_{n}\right) \rightarrow g(\bar{x})$. ( $g$ is continuous as it is Fréchet differentiable.) It follows that $g^{\prime}(\bar{x})(h) \in T_{\tau_{2}}(B, g(\bar{x}))$.

Since $g^{\prime}(\bar{x})$ is continuous and linear, it preserves the closed convex hulls of sets and $g^{\prime}(\bar{x})\left(P_{\tau_{1}}(\Delta, \bar{x})\right) \subset P_{\tau_{2}}(B, g(\bar{x}))$.

Corollary 1. If $g^{\prime}(\bar{x})$ is compact then $g^{\prime}(\bar{x})\left(P_{\sigma}(\Delta, \bar{x})\right) \subset P(B, g(\bar{x}))$.

Remarks. (i) Note that $g^{\prime}(\bar{x})$, being a Fréchet derivative, is always $s-s$ continuous and so, being linear, is always $\sigma-\sigma$ continuous. Similarly if $X$ and $Y$ are dual spaces and $g^{\prime}(\bar{x})$ is an adjoint map it is $\sigma^{*}-\sigma^{*}$ continuous.

(ii) These results hold true in locally convex spaces if one uses bounded derivatives ([1]). This remains true for the rest of the development. If one is interested only in strong cones one can replace Fréchet derivatives by Gateaux derivatives of locally Lipshitz functions.

The next result generalizes Theorem 1 of Guignard [12].

TheOREM 1. Suppose that $f^{\prime}(\bar{x})$ is $\tau$-continuous. A necessary condition for $\bar{x}$ to minimize fover $A$ is that $f^{\prime}(\bar{x}) \in P_{\tau}(A, \bar{x})^{+}$. If $f$ is pseudoconvex over $A$ at $\bar{x}$ and $A$ is $\tau-$ pseudoconvex at $x_{0}$, then the condition is also sufficient.

Proof. (i) Necessity: Suppose that $h \in T_{\tau}(A, \bar{x})$. As in Proposition 6 there is a sequence $\left\{x_{n}\right\}, x_{n} \in A, x_{n} \rightarrow \bar{x}$ and a sequence of positive real numbers $\left\{\lambda_{n}\right\}$ with $h_{n}=$ $\lambda_{n}\left(x_{n}-\bar{x}\right)$ and $h_{n} \rightarrow h$. Again, as in Proposition 6,

$$
\lambda_{n}\left(f\left(x_{n}\right)-f(\bar{x})\right)=\lambda_{n}\left(f\left(\bar{x}+\lambda_{n}^{-1} h_{n}\right)-f(\bar{x})\right) \rightarrow f^{\prime}(\bar{x})(h),
$$

because $f^{\prime}(\bar{x})$ is assumed $\tau$-continuous. By assumption $x_{n} \in A$ so that $f\left(x_{n}\right) \geqq f(\bar{x})$. Since $\lambda_{n} \geqq 0$, one has $f^{\prime}(\bar{x})(h) \geqq 0$. This holds for all $h \in T_{\tau}(A, \bar{x})$. By continuity and linearity of $f^{\prime}(\bar{x})$, one has in fact $f^{\prime}(\bar{x}) \in P_{\tau}(A, \bar{x})^{+}$.

(ii) Sufficiently: Suppose $f^{\prime}(\bar{x}) \in P_{\tau}(A, \bar{x})^{+}$. Then $f^{\prime}(\bar{x})(h) \geqq 0$ for all $h$ in $P_{\tau}(A, \bar{x})$. Since $A$ is $\tau$-pseudoconvex at $\bar{x}, f^{\prime}(\bar{x})(x-\bar{x}) \geqq 0$ for all $x \in A$. This in turn implies that $f(x) \geqq f(\bar{x})$ for $x \in A$, because $f$ is supposed pseudoconvex. Thus $\bar{x}$ minimizes $f$ over A.

When $\tau$ is a topology of the dual pair $f^{\prime}(\bar{x})$ is always $\tau$-continuous. If $X$ is a dual space $E$ the condition that $f^{\prime}(\bar{x})$ is $\sigma^{*}$-continuous requires that $f^{\prime}(\bar{x}) \in E$ not only that $E^{\prime \prime}=X^{\prime}$. By Corollary 5 of [2] this is true if $f$ is a $\sigma^{*}$-lower semicontinuous convex function which is Fréchet differentiable at $\bar{x}$.

Guignard proved Theorem 1 with $P_{\tau}(A, \bar{x})$ replaced by $P(A, \bar{x})$ and, in the sufficiency proof with $A$ assumed pseudoconvex. Since $P(A, \bar{x}) \subset P_{\tau}(A, \bar{x})$, it is immediate that Theorem 1 is more general. Moreover, as the next example shows, in some cases Theorem 1 gives more information. 
Example 2. (a) Let $X=l_{2}(N)$ and $A=\left\{\left(e_{n}+e_{1}\right) / n\right\}_{n=2}^{\infty} \cup\{0\}$, where $e_{n}$ is the sequence with a one in the $n$th position and zeros elsehwere. Suppose $f: X \rightarrow R$ bas a minimum at 0 over $A$. Now $T(A, 0)=P(A, 0)=0$ while $e_{1} \in T_{\sigma}(A, 0) \subset P_{\sigma}(A, 0)$. Thus Theorem 1 implies that $f^{\prime}(0)\left(e_{1}\right) \geqq 0$ while Theorem 1 in [12] gives no information.

(b) Now consider the same example with $X=l_{1}(N)$. Again $T(A, 0)=P(A, 0)=0$ while now $T_{\sigma}(A, 0)=0$ since weak and strong sequential convergence agree in $l_{1}(N)$. By considering $X=c_{0}^{\prime}$ we can verify that in $\sigma^{*}=\sigma\left(l_{1}, c_{0}\right)$ we have $e_{1} \in T_{\sigma^{*}}(A, 0)$. Thus if $f^{\prime}(\bar{x}) \in c_{0}$ we can get information from Theorem 1 again even though weak tangent cones provide no information.

Let $K(\tau)=\left\{h \in X: g^{\prime}(\bar{x})(h) \in P_{\tau}(B, g(\bar{x}))\right\}$ and let $H(\tau)=\left\{h^{\prime} \in X^{\prime}: h^{\prime}=u^{+} g^{\prime}(\bar{x})\right.$, $\left.u^{+} \in P_{\tau}(B, g(\bar{x}))^{+}\right\}$.

Proposition 7. $K(\tau)^{+}=\overline{H(\tau)}$. In addition when one has $R\left(g^{\prime}(\bar{x})\right) \cap$ $P_{\tau}(B, g(\bar{x}))^{0} \neq \varnothing$, then $K(\tau)^{+}=H(\tau)$.

Proof. Suppose $h \in K(\tau)$ and $h^{\prime} \in H(\tau)$. Then $h^{\prime}=u^{+} g(\bar{x})$, with $u^{+} \in P_{\tau}(B, g(\bar{x}))$, and $h^{\prime}(h)=\left(u^{+} g^{\prime}(\bar{x})\right)(h)=u^{+}\left(g^{\prime}(\bar{x})(h)\right) \geqq 0$. Thus $K(\tau) \subset H^{+}(\tau)$. If $h_{1} \notin K(\tau)$, there is, by the strong separation theorem, some $u^{+} \in P_{\tau}(B, g(\bar{x}))^{+}$with $u^{+} g^{\prime}(\bar{x})\left(h_{1}\right)<0$. Thus $h_{1} \notin H^{+}(\tau)$ and $H^{+}(\tau)=K(\tau)$. Proposition 1(ii) shows that $K^{+}(\tau)=H^{++}(\tau)=\overline{H(\tau)}$. Suppose that the auxiliary condition is met.

Theorem 3 of $[15$, p. 5] guarantees that in a locally convex setting

$$
\partial f(x)=T^{*} \partial d(T x)
$$

where $d$ is a convex function, $T$ is a continuous linear operator and $f(x)=d(T x)$. For (7) to hold one needs $d$ continuous at some point of $R(T)$. One quickly verifies that this is true for $d(y)=i\left(y \mid P_{\tau}(B, g(\bar{x}))\right)$ and $T=g^{\prime}(\bar{x})$. Apply (7) with $x=0$ and one has (see the discussion below Definition 3 )

$$
K(\tau)^{-}=\partial f(0)=g^{\prime}(\bar{x})^{*} \partial d(0)=g^{\prime}(\bar{x})^{*} P_{\tau}(B, g(\bar{x}))^{-},
$$

which clearly yields the desired equivalence.

Proposition 7 gives conditions which exclude the example given by Zlobec in [27] which exhibits a case in which $H$ is not closed but $R\left(g^{\prime}(\bar{x})\right)$ is. In his example the interior condition is violated.

THEOREM 2 (The generalized Kuhn-Tucker conditions). Suppose that $H\left(\tau_{2}\right)$ is closed and that $G \subset X$ is a closed convex cone with $K\left(\tau_{2}\right) \cap G \subset P_{\tau_{1}}(A, \bar{x})$. Suppose that $K^{+}\left(\tau_{2}\right)+G^{+}$is closed. A necessary condition for $\bar{x}$ to minimize (P) when $f^{\prime}(\bar{x})$ is $\tau_{1}$-continuous is that there exist $u^{+} \in P_{\tau_{2}}(B, g(\bar{x}))^{+}$with

$$
f^{\prime}(\bar{x})-u^{+} g^{\prime}(\bar{x}) \in G^{+} \text {. }
$$

This condition is also sufficient if (i) $G$ is a closed convex cone containing $A-\bar{x}$, (ii) $g^{\prime}(\bar{x})$ is $\tau_{1}-\tau_{2}$ continuous, (iii) $A$ is $\tau_{1}$-pseudoconvex with respect to $\Delta$ at $\bar{x}$, and if (iv) $f$ is pseudoconvex over $A$ at $\bar{x}$.

Proof. (i) Necessity: By Theorem $1 f^{\prime}(\bar{x}) \in P_{\tau_{1}}(A, \bar{x})^{+}$. Since $K\left(\tau_{2}\right)^{+}+G^{+}$is assumed closed and $K\left(\tau_{2}\right) \cap G \subset P_{\tau_{2}}(A, \bar{x})$ one has

$$
f^{\prime}(\bar{x}) \in \overline{K\left(\tau_{2}\right)^{+}+G^{+}}=K\left(\tau_{2}\right)^{+}+G^{+} .
$$

This uses Proposition 1(ii). Proposition 7 and the assumption that $H\left(\tau_{2}\right)$ is closed mean that $f^{\prime}(\bar{x}) \in P_{\tau_{1}}(A, \bar{x})^{+} \subset H\left(\tau_{2}\right)+G^{+}$. This is the required result.

(ii) Sufficiency: Since $A-\bar{x} \subset G$ one has

$$
f^{\prime}(\bar{x})(x-\bar{x}) \geqq u^{+} g^{\prime}(\bar{x})(x-\bar{x}) \quad \forall x \in A \text {. }
$$


Since $A-\bar{x} \subset P_{\tau_{1}}(\Delta, \bar{x})$ by assumption, and since $g^{\prime}(\bar{x})\left(P_{\tau_{1}}(\Delta, \bar{x})\right)$ is contained in $P_{\tau_{2}}\left(B, g((\bar{x}))\right.$ by Proposition $6, g^{\prime}(\bar{x})(x-\bar{x}) \in P_{\tau_{2}}(B, g(\bar{x}))$ for all $x$ in $A$. Since $u^{+} \in$ $P_{\tau_{2}}(B, g(\bar{x}))^{+}$, this implies that $f^{\prime}(\bar{x})(x-\bar{x}) \geqq 0$ for all $x$ in $A$. Because $f$ is assumed pseudoconvex over $A, f(x) \geqq f(\bar{x})$ for $x$ in $A$.

Let $H\left(G, \tau_{1}, \tau_{2}\right)$ denote the hypotheses that $K^{+}\left(\tau_{2}\right)+G^{+}$and $H\left(\tau_{2}\right)$ are $\sigma\left(X^{\prime}, X\right)$ closed with $K\left(\tau_{2}\right) \cap G \subset P_{\tau_{1}}(A, \bar{x})$.

COROLlARY 2. $H\left(G, \tau_{1}, \tau_{2}\right)$ is satisfied when $G \cap K\left(\tau_{2}\right) \subset P_{\tau_{1}}(A, \bar{x})$ and

(i) $G^{0} \cap K\left(\tau_{2}\right) \neq \varnothing$ or $G \cap K\left(\tau_{2}\right)^{0} \neq \varnothing$,

(ii) $\left(R g^{\prime}(\bar{x})\right) \cap P_{\tau_{2}}(B, g(\bar{x}))^{0} \neq \varnothing$.

Proof. This follows from Proposition 2 and Proposition 7.

THEOREM 2'. The following conditions are also sufficient in Theorem 2:

(i) $A-\bar{x} \subset G$,

(ii) $g(A)$ is $\tau_{2}$-pseudoconvex with respect to $B$ at $g(\bar{x})$,

(iii) the function $f-u^{+} g$ is pseudoconvex over $A$ at $\bar{x}$.

Proof. As before we have

$$
\left(f^{\prime}(\bar{x})-u^{+} g^{\prime}(\bar{x})\right)(x-\bar{x}) \geqq 0 \quad \forall x \in A .
$$

Since $f-u^{+} g$ is assumed pseudoconvex at $\bar{x}$ we have

$$
f(x)-u^{+} g(x) \geqq f(\bar{x})-u^{+} g(\bar{x}) \geqq 0 \quad \forall x \in A .
$$

Or

$$
f(x)-f(\bar{x}) \geqq u^{+}(g(x)-g(\bar{x})) \quad \forall x \in A .
$$

Hypothesis (ii) implies that $g(x)-g(\bar{x}) \subset P_{\tau_{2}}(B, g(\bar{x}))$ for each $x \in A$. As $u^{+} \in$ $P_{\tau_{2}}(B, g(\bar{x}))^{+},(8)$ implies that $\bar{x}$ minimizes $f$ over $A$.

Remarks. (i) Hypothesis (ii) is guaranteed when $B$ is convex while hypothesis (iii) is certainly weaker than asking for $f$ to be convex and $g$ to be concave. Indeed simple examples can be created to show that (ii) and (iii) are not subsumed in Theorem 2 partially because these conditions make use of $u^{+}$which the previous ones do not.

(ii) Example 1 and the discussion preceeding it shows that weak pseudoconvexity is a good deal less restrictive a property than pseudoconvexity.

In finite dimensions polyhedralness of the various cones suffices to guarantee closure in the corollary. Guignard has indicated in [12] how $H\left(G, \tau_{1}, \tau_{2}\right)(H(G)$ in her case) includes the standard Kuhn-Tucker conditions in finite dimensions where all linear topologies agree. It is also easy to verify that the standard infinite dimensional "constraint qualifications" are subsumed.

The asymptotic results of Zlobec [27] and Zlobec and Massam [28] can also be proved correspondingly for $\tau$-tangent cones, as can the Pareto optimum results in [5]. Proposition 4 can be used to give conditions under which $P_{\tau}(A, \bar{x})=$ $P_{\tau}(\Delta, \bar{x}) \cap P_{\tau}(C, \bar{x})$. This, in conjunction with the requirement that $P_{\tau}(\Delta, \bar{x})=K(\tau)$, gives conditions for Theorem 2 to hold with $G=P_{\tau}(C, \bar{x})$. In particular, $P(\Delta, \bar{x})=K$ when $B$ is a closed convex cone with interior and $g^{\prime}(\bar{x})(\bar{h})+g(\bar{x}) \in B^{0}$ for some $\bar{h} \in X$. This last condition also guarantees that Proposition 7 applies and $H(\tau)$ is closed.

4. Fritz John conditions. In the case that $H\left(G, \tau_{1}, \tau_{2}\right)$ does not hold one can still often give a necessary condition for optimality. Such a condition is generally called a Fritz John condition [16]. The theorem stated below is the $\tau$-tangent cone analogue of one in Nagahisa and Sakawa [20].

THEOREM 3. Suppose that $\bar{x}$ is minimal for (P) with $B$ a closed convex cone with interior. Suppose $f^{\prime}(x)$ is $\tau$-continuous and $g^{\prime}(\bar{x})$ is $\tau$-s continuous. For any closed convex 
cone $M$ in $T_{\tau}(C, \bar{x})$ there exist $r^{+} \geqq 0$ and $u^{+} \in B^{+}$with $u^{+} g(\bar{x})=0$, not both zero, such that

$$
r^{+} f^{\prime}(\bar{x})-u^{+} g^{\prime}(\bar{x}) \in M^{+} \text {. }
$$

Proof. As in [20], consider $S_{1}=\left\{(r, z) \mid f^{\prime}(\bar{x})(h) \leqq r, g^{\prime}(\bar{x})(h)+g(\bar{x})-z \in B\right.$, for some $h \in M\}$ and $S_{2}=\{(r, z) \mid r \leqq 0, z \in B\}$. It suffices to show that $S_{1}$ and $S_{2}$ are convex sets with $S_{1} \cap S_{2}^{0}=\varnothing$ since then they can be separated and the separating hyperplane $z^{+}=\left(r^{+}, u^{+}\right)$has the requisite properties exactly as in [20]. Suppose, therefore, that $S_{1} \cap S_{2}^{0} \neq \varnothing$. There is then some $\bar{h} \in M$ with $f^{\prime}(\bar{x})(\bar{h})<0$ and $g^{\prime}(\bar{x})(\bar{h})+g(\bar{x}) \in B^{0}$. Since $\bar{h} \in M$, there is a sequence $\left\{x_{n}\right\}$ in $C$ with $x_{n} \rightarrow \bar{x}$, and $\lambda_{n} \subset R^{+}$with $h_{n}=$ $\lambda_{n}\left(x_{n}-\bar{x}\right) \rightarrow(\tau) \bar{h}$. Now $g^{\prime}(\bar{x})$ is $\tau-s$ continuous, so that there exists some $n_{0} \in N$ such that $g^{\prime}(\bar{x})\left(h_{n}\right)+g(\bar{x}) \in B^{0}$ for $n \geqq n_{0}$. Similarly, for $n \geqq n_{1}, f^{\prime}(\bar{x})\left(h_{n}\right)<0$. It follows from (5) of Proposition 6 that for $n$ large enough one has

$$
\lambda_{n}\left(g\left(\bar{x}+\lambda_{n}^{-1} h_{n}\right)-g(\bar{x})\right)+g(\bar{x}) \in B^{0} .
$$

$\lambda_{n}$ is positive so that

$$
g\left(x_{n}\right) \in B^{0}+\left(1-\lambda_{n}^{-1}\right) g(\bar{x}) .
$$

Since $\lambda_{n}^{-1} \rightarrow 0$ and $g(\bar{x}) \in B$ one must have $g\left(x_{n}\right) \in B^{0}+B \subset B^{0}$ for $n$ sufficiently large. A similar argument shows that $f\left(x_{n}\right)<f(\bar{x})$ for large $n$. Since $x_{n} \in C$ this contradicts the optimality of $\bar{x}$. Thus $S_{1} \cap S_{2}^{0}=\varnothing$. Since both $S_{1}$ and $S_{2}$ are convex they can be separated and the proof proceeds as in [20].

Remark. (i) The requirement that $g^{\prime}(\bar{x})$ be $\tau$-s continuous can be replaced by the condition that $g^{\prime}(\bar{x})$ be $\tau-\tau_{2}$ continuous and $B$ have interior in the $\tau_{2}$ topology as one can still separate $S_{1}$ and $S_{2}$ in that case.

(ii) If $\tau$ is a dual topology one can require that $g^{\prime}(\bar{x})$ is compact, or, of course, finite dimensional.

As a consequence of this result one can extend the theorems in Craven [8] and Craven and Mond [9] on Fritz John conditions with equality constraints. Two definitions are necessary.

Definition 12 [8]. A continuous linear map $B: X \rightarrow Z$ is said to be adequate if (i) $R(B)$ is closed in $Z$, and if (ii) $R(B)=Z$ then there is a continuous projection of $X$ onto $N(B)$.

DEFINITION 13. Let $h: X \rightarrow Z$ be a Fréchet differentiable map between two Banach spaces. $h$ will be called $(G, \tau)$-regular at $\bar{x}$ when $R\left(h^{\prime}(x)\right)$ is closed and when $R\left(h^{\prime}(x)\right)=Z$ implies $N\left(h^{\prime}(x)\right) \cap G \subset T_{\tau}(N(h), \bar{x})$ for some closed convex cone $G$ with $G^{0} \cap N\left(h^{\prime}(\bar{x})\right) \neq \varnothing$.

In particular, it follows, from Flett's results in [10], that a continuously Fréchet differentiable map with a surjective derivative at $\bar{x}$ is $(G, \tau)$-regular for $G=X, \tau=s$. In general $(G, \tau)$-regularity caters to the possibility that $g^{\prime}(x)$ is not continuous in $x$.

THEOREM 4. Suppose that in Theorem $3 C=N(h)$ where $h$ is $(G, \tau)$-regular at $\bar{x}$. Then there exist $r^{+} \geqq 0, u^{+} \in B^{+}, z^{+} \in Z^{\prime}$, not all zero, such that $r^{+} f^{\prime}(\bar{x})-u^{+} g^{\prime}(\bar{x})-$ $z^{+} h^{\prime}(\bar{x}) \in G^{+} ; u^{+} g(\bar{x})=0$. Moreover, when $h^{\prime}(\bar{x})$ is surjective, one of $u^{+}, r^{+}$can be

Proof. In the case that $\overline{R\left(h^{\prime}(\bar{x})\right)} \neq Z$ one can find some nonzero $z^{+} \in Z^{\prime}$ with $z^{+} h^{\prime}(\bar{x})=0$. Setting $u^{+}=0$ and $r^{+}=0$, one is done. Suppose now that $R\left(h^{\prime}(\bar{x})\right)=Z$. Then $N\left(h^{\prime}(\bar{x})\right) \cap G \subset T_{\tau}(N(h), \bar{x})$. Since $N\left(h^{\prime}(\bar{x})\right) \cap G$ is a closed convex cone in $T_{\tau}(C, \bar{x})$, (with $C=N(h)$ ) one can apply Theorem 3 to derive that $r^{+} \geqq 0, u^{+} \in B^{+}$with $u^{+} g(\bar{x})=0$ exist such that $r^{+} f^{\prime}(\bar{x})-u^{+} g^{\prime}(\bar{x}) \in\left(N\left(h^{\prime}(\bar{x})\right) \cap G\right)^{+}$. Since $G^{0} \cap N\left(h^{\prime}(\bar{x})\right) \neq$ 
by hypothesis this can be rewritten as

$$
r^{+} f^{\prime}(\bar{x})-u^{+} g^{\prime}(\bar{x}) \in N\left(h^{\prime}(\bar{x})\right)+G^{+}
$$

A simple application of the Farkas lemma [15] shows that any member $y^{+}$of $N\left(h^{\prime}(\bar{x})\right)^{+}$can be written as $z^{+} h^{\prime}(\bar{x})$ with $z^{+} \in Z^{\prime}$. This concludes the proof.

Craven and Mond's Theorem 4 in [9] is essentially a corollary of the present Theorem 4 and $G=X$. There it is proved for an adequate continuously differentiable $h$. As has been seen such a mapping is $G$-regular with $G=X$. This theorem is in itself a generalization of the Fritz John results in [16]. Note that adequacy is entirely too strong a notion.

Zlobec and Massam state Theorem 3 for (strong) tangent cones in locally convex spaces with an erroneous formulation. Theorem 3 of this paper can also be extended to locally convex spaces with bounded differentiation.

We conclude this section by observing that the condition in Theorem 3 and 4 that $B$ be a cone can be removed as follows:

THEOREM 5. Assume the hypotheses of Theorem 3 save that $B$ need now only be a closed convex set with interior. Then the conclusions hold except that now

$$
u^{+} \in(B-g(\bar{x}))^{+}=P(B, g(\bar{x}))^{+} .
$$

Proof. Consider $g_{1}: X \rightarrow Y \times R$ given by $g_{1}(x)=(g(x), 1)$. Let $S=\{(x, r): x \in r B$, $r \geqq 0\}$. We can check that

$$
g(x) \in B \Leftrightarrow g_{1}(x) \in \bar{S} .
$$

Moreover $\bar{S}$ is a closed convex cone with interior and so we may apply Theorem 3 to deduce that

$$
r^{+} f^{\prime}(\bar{x})-s^{+} g_{1}^{\prime}(\bar{x}) \in M^{+}, \quad s^{+} g_{1}(\bar{x})=0
$$

with $r^{+} \geqq 0, s^{+} \in S^{+}$not both zero. By construction of $S, s^{+}=\left(b^{+}, r_{1}^{+}\right)$with

$$
b^{+}(b)+r_{1}^{+} \geqq 0 \quad \forall b \in B .
$$

Also

$$
b^{+} g(\bar{x})+r_{1}^{+}=s^{+} g_{1}(\bar{x})=0 .
$$

Together (10) and (11) show that $b^{+} \in(B-g(\bar{x}))^{+}$. From (11) it also follows that $s^{+} \neq 0$ implies $b^{+} \neq 0$. Finally, since

$$
s^{+} g_{1}^{\prime}(\bar{x})=b^{+} g^{\prime}(\bar{x})+r_{1}^{+} 0=b^{+} g^{\prime}(\bar{x}),
$$

we obtain the desired conclusion by substituting (12) into (9).

Applications. We do not give an application of these results here. However, Theorem 4 can be used to give an analogous application to optimal control to that given by Craven and Mond [9]. One merely relaxes their condition of adequacy to $G$-regularity. Similar remarks apply to the Kuhn-Tucker theorems for which examples (mainly finite dimensional) are given in [12].

5. On the "necessity" of the constraint qualification. Various technical results are possible when $\tau_{1}$ is a topology which makes the unit ball $B(X)$ in $X$ a compact set. It is known in this case that $X=E^{\prime}$ where $E$ is a Banach space [14]. For simplicity we 
confine ourselves to the case in which $X$ is reflexive and $\tau_{1}=\sigma\left(X, X^{\prime}\right)$. As in [4] we let

$$
\begin{aligned}
& F(A, \bar{x})=\{f: f \text { is Fréchet differentiable at } \bar{x} \text { and achieves }\text { a (local) minimum over } A \text { at } \bar{x}\}, \\
& M(A, \bar{x})=\left\{f^{\prime}(\bar{x}): f \in F(A, \bar{x})\right\} .
\end{aligned}
$$

It is proved in [4], that when $X$ is reflexive

$$
P_{\sigma}(A, \bar{x})^{+} \subset M(A, \bar{x}) .
$$

(An inspection of the proof of (13) in [4] shows that if $X=E^{\prime}$ and $\tau_{1}$ makes $B(X)$ sequentially compact one in fact has $\left.P_{\tau_{1}}(A, \bar{x})^{+} \cap E \subset M(A, \bar{x}) \cap E\right)$.

Consider the special case of $(\mathrm{P})$ in which $C=X$.

$$
\min \{f(x): g(x) \in B\} .
$$

THEOREM 6. Suppose $X$ is reflexive and $g^{\prime}(x)$ is $\sigma-\tau$ continuous and that

$$
f^{\prime}(\bar{x})-u^{+} g^{\prime}(\bar{x})=0 ; \quad u^{+} \in P_{\tau}(B, g(\bar{x}))^{+}
$$

holds for all $f \in F(A, \bar{x})$. Then $H(X, \sigma, \tau)$ holds. In other words the constraint qualification is necessary in this case.

Proof. From (13) it follows that $M(A, \bar{x}) \subset H(\tau)$. Now

$$
H(\tau) \subset K(\tau)^{+} \subset P_{\sigma}(A, \vec{x})^{+},
$$

where the first inequality follows from Proposition 7 and the second from Proposition 6. Since (14) holds we have

$$
P_{\sigma}(A, \bar{x})^{+}=H(\tau)=K(\tau)^{+}
$$

which is $H(X, \sigma, \tau)$.

This extends the corresponding results in [4], from the case in which $B$ is the orthant in $R^{n}$. A moment's reflection on Example 2(b) and Theorem 1 shows that (13) fails in a more general dual space since we may have $P_{\sigma}(A, \bar{x})^{+}=X^{\prime}$ while $P_{\sigma^{*}}(A, \bar{x})^{+} \neq X^{\prime}$. The latter will serve as a constraint on at least some members of $M(A, \bar{x})$. One might hypothesize that (13) in fact characterizes reflexive spaces. In any case the general question of necessity of $H\left(G, \tau_{1}, \tau_{2}\right)$ still remains open even for $G=X$ and $\tau_{1}=\sigma^{*}$. It would also be interesting to characterize the existence of a bounded or compact set of multipliers along the lines of the work in [11].

Theorem 6 thus illustrates another reason for examining $\tau$-tangent cones. Clarke [7] has defined and exploited a different tangent cone $T_{E}(x)$. It agrees with this notion on convex sets but it is always convex and satisfies $T_{E}(x) \subset T(E, x)$. Thus in general his normal cone $N_{E}(x)=T_{E}(x)^{+}$is strictly larger than $T(E, x)^{+}$. It therefore constrains a derivative less to belong to $N_{E}(x)$ than to $T_{E}(x)^{+}$. Clarke's optimal condition applies to all generalized derivatives [7] while this tangent cone approach requires something close to Fréchet derivatives to exist. The author intends to systematically explore the comparisons between the two cone notions in an upcoming paper.

Acknowledgments. I would like to thank Dr. M. A. H. Dempster, Dr. R. O'Brien and the referee for their generous suggestions.

\section{REFERENCES}

[1] J. M. ABADIE, Problémes d'optimisation, Institut Blaise Pascal, Paris, 1965.

[2] E. Asplund AND R. T. RockAfEllar, Gradients of convex functions, Trans. Amer. Math. Soc., 139 (1969), pp. 443-467. 
[3] V. T. Averbukh And O. G. Smolyanov, The various definitions of the derivative in linear topological spaces, Russian Math. Surveys, 23 (1968), no. 4, pp. 67-114.

[4] M. S. BAZARAA, J. J. GoOde, M. F. NASHEd AND C. M. SHETTY, Nonlinear programming without differentiability in Banach Spaces. Necessary and sufficient constraint qualifications, J. Appl. Analysis, 5 (1976), pp. 165-173.

[5] J. BORWEIN, Proper efficient points for maximizations with respect to cones, this Journal, 15 (1977), pp. 57-63.

[6] J. BORWEIN AND R. O'Brien, Tangent cones and convexity, Canad. Math. Bull., 19 (1976), pp. 257-261.

[7] F. H. Clarke, Generalized gradients and applications, Trans. Amer. Math. Soc., 205 (1975), pp. 247-262.

[8] B. D. CRAVEN, Nonlinear programming in locally convex spaces, J. Optimization Theory Appl., 10 (1970), pp. 197-210.

[9] B. D. CRAVEN AND B. MOND, Transposition theorems for cone-convex functions, SIAM J. Appl. Math., 24 (1973), pp. 603-612.

[10] T. M. FLETT, On differentiation in normed vector spaces, J. London Math. Soc., 42 (1967), pp. 523-533.

[11] J. GAUVIN, A necessary and sufficient regularity condition to have bounded multipliers in nonconvex programming, Math. Programming, 12 (1977), pp. 136-138.

[12] M. GuIGNARD, Generalized Kuhn-Tucker conditions for mathematical programming in a Banach space, this Journal, (1969), pp. 232-241.

[13] H. HALKIN, Implicit functions and optimization problems without continuous differentiability of the data, C.O.R.E. Discussion Paper no. 7206, Centre for Operations Research and Econometrics, (1972).

[14] R. B. Holmes, Geometric Functional Analysis and its Applications, Springer-Verlag, New York, 1975).

[15] A. D. IOFFE AND V. L. Levin, Differentials of convex functions, Trans. Moscow Math. Soc., 26 (1972), pp. 1-71.

[16] F. JOHN, Extremum problems with inequalities as subsidiary conditions, Studies and Essays, Courant Anniversary Volume, Interscience, New York, 1948, pp. 187-204.

[17] H. W. KUHN AND A. W. TUCKER, Nonlinear programming, Proc. Second Berkeley Symposium on Math. Statistics and Probability, J. Neyman, ed., University of California Press, Berkeley, CA, 1951.

[18] Ka-Sing LAU, Almost Chebyshev subsets in reflexive Banach spaces, to appear.

[19] O. L. MANGASARIAN AND S. FROMOVITZ, The Fritz John necessary optimality conditions in the presence of equality and inequality constraints, J. Math. Anal. Appl., 17 (1967), pp. 37-47.

[20] Y. NAGAHISA AND Y. SAKAWA, Nonlinear programming in Banach spaces, J. Optimization Theory Appl., 4 (1969), pp. 182-190.

[21] K. RITTER, Optimization in linear spaces I, II, III, Math. Ann., 182 (1969), pp. 189-206, 183 (1969), pp. 169-180.

[22] A. P. AND W. J. Robertson, Topological Vector Spaces, Cambridge University Press, London, 1964.

[23] R. T. RockAfellar, An extension of Fenchel's duality theorem for convex functions, Duke Math. J., 33 (1966), pp. 81-90.

[24] - Convex Analysis, Princeton University Press, Princeton, NJ, 1970.

[25] P. P. VARAIYA, Nonlinear programming in Banach spaces, SIAM J. Appl. Math., 19 (1967), pp. 239-244.

[26] S. YAmamuro, Differential Calculus in Topological Linear Spaces, Springer-Verlag, New York, 1974.

[27] S. ZlOBEC, Asymptotic Kuhn-Tucker conditions for mathematical programming problems in Banach space, this Journal, 8 (1970), pp. 505-512.

[28] S. ZLOBEC AND H. MASSAM, Various definitions of the derivative in mathematical programming, Math. Programming, 7 (1974), pp. 144-161. 